\title{
Artigo \\ Aperfeiçoamento de algoritmos de sincronização de registros oscilográficos baseados em de tensão e corrente pré-falta
}

\author{
Jackson Nogueira Feitosa ${ }^{[1]}$, Melinda Cesianara Silva da Cruz ${ }^{[2]}$ \\ ${ }^{[1]}$ Universidade Federal Rural do Semi-árido; jacksonfeitosa16@gmail.com \\ ${ }^{[2]}$ Universidade Federal Rural do Semi-árido; melinda@ufersa.edu.br \\ Recebido: 10/07/2019; \\ Aceito: $12 / 07 / 2019$; \\ Publicado: 07/10/2019.
}

Resumo: Algoritmos de sincronização de registros oscilográficos são ferramentas importantes para a operação de sistemas de potência, sendo capazes de determinar o ângulo de correção necessário para alimentar, por exemplo, softwares de localização de faltas em linhas de transmissão e distribuição de energia. Existem na literatura vários métodos que fazem a sincronização de registros, sendo que os métodos mais vantajosos são aqueles que independem das características elétricas da linha como resistência, indutância e capacitância, as quais podem conter erros. Este trabalho propõe realizar uma análise e sugerir melhorias na implementação de duas metodologias de sincronização de registros oscilográficos existentes na literatura, ambas baseadas em fasores de tensão e corrente pré-falta, e independentes dos parâmetros elétricos da linha. Nos testes efetuados foram utilizados fasores de tensão e corrente obtidos a partir de simulações realizadas nos softwares ATPDraw e ANAREDE.

Palavras-chave: Sincronização de registros oscilográficos; localização de faltas; linhas de transmissão; parâmetros elétricos.

\section{INTRODUÇÃO}

São encontrados na literatura diversos estudos sobre a localização de faltas em linhas de transmissão

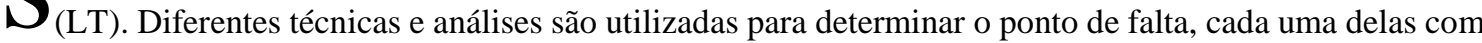
suas vantagens e desvantagens. Porém, essas técnicas, em sua maioria, necessitam de mecanismos capazes de sincronizar dados de tensão e corrente dos dois terminais da linha [1].

Relata-se em [2], que diversos estudiosos admitem empregar em suas análises, dados de tensão e corrente sincronizados, previamente, por PMUs (Phasor Measurement Units). PMUs são equipamentos que medem grandezas fasoriais utilizando o sistema de GPS (Global Positioning System) necessitando de um grande sistema de comunicação, o qual possui custos elevados. Inclusive, perdas no sinal de GPS podem acarretar em uma má sincronização de registros dos dados. Dessa forma, a sincronização de dados realizada via software ainda é um instrumento importante para auxiliar na extração correta de fasores necessários para determinar o local de uma falta em uma linha de transmissão.

Grande parte dos algoritmos que realizam a sincronização de registros de falta levam em consideração as componentes transitórias da linha de transmissão, destacando-se os fundamentados pelo uso da Transformada Walvelet [3] e algoritmos que comparam, em um sinal amostrado, valores instantâneos de dois ciclos consecutivos [4]. Encontram-se na literatura ainda técnicas que utilizam fasores de tensão e corrente pré-falta, dependentes dos parâmetros elétricos da linha [5], e não dependentes desses parâmetros [1-2]. Vale salientar que os parâmetros elétricos das linhas de transmissão podem conter erros ou podem estar indisponíveis 
computacionalmente, sendo relevante o uso de ferramentas que independam desses dados, uma vez que isso significa a exclusão de uma possível fonte de erros.

Este artigo apresenta uma modificação nas metodologias apresentadas em [1] e [2] para a determinação do ângulo de sincronização de registros, visto que o primeiro algoritmo apresentou falhas em algumas situações de operação do sistema e o segundo apresenta um número excedente de condições e equações para a determinação do ângulo correto de sincronização. Portanto, o novo algoritmo que vêm a ser proposto neste trabalho, resultante do aperfeiçoamento dos métodos descritos em [1] e [2], continua identificando os ângulos de sincronização em qualquer quadrante, e apresentando o benefício de operar corretamente em qualquer condição de operação do sistema elétrico com um menor número de condições a serem atendidas.

O procedimento sugerido bem como as duas metodologias analisadas, independe de parâmetros elétricos e características transitórias da linha de transmissão, pois o mesmo necessita apenas dos fasores de tensão e corrente pré-falta. Inclusive, a metodologia por depender destes dados pode ser aplicada em casos de difícil sincronização, onde existam parâmetros de linha desconhecidos ou inexatos, e registros com características transitórias atípicas [1]. Neste estudo foi realizada uma análise de desempenho da metodologia proposta com base em dados simulados no software ATPDraw (Alternative Transient Program) e no software ANAREDE (Análise de Redes Elétricas).

\section{ALGORITMOS DE SINCRONIZAÇÃO DE REGISTROS DE FALTA ANALISADOS}

A seguir será apresentado o equacionamento que constitui o algoritmo de sincronização de registros oscilográficos descrito em [1]. Para isso, considerar a Figura 1, que mostra o circuito equivalente de sequência positiva de uma linha de transmissão no instante pré-falta.

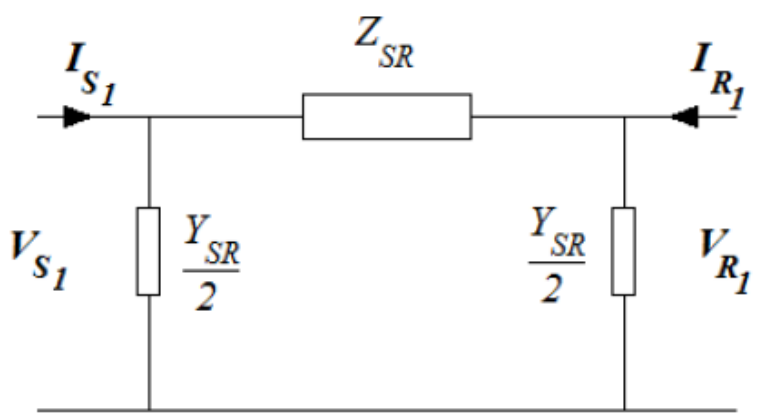

FIGURA 1. Circuito equivalente de sequência positiva de uma LT no instante pré-falta. [1]

Na Figura 1, os termos $Z_{S R}$ e $Y_{S R}$ representam, respectivamente, a impedância série e admitância shunt de sequência positiva da LT. Os termos $V_{S_{1}}, I_{S_{1}}, V_{R_{1}}, I_{R_{1}}$, representam, nesta ordem, os fasores de tensão e corrente pré-falta nos terminais $\mathrm{S}$ e $\mathrm{R}$, como também o índice 1 diz respeito à sequência positiva da linha de transmissão.

Da análise da Figura 1, obtém-se a Equação (1).

$$
\mathrm{I}_{\mathrm{S}_{1}}+\mathrm{I}_{\mathrm{R}_{1}} \mathrm{e}^{\mathrm{j} \delta}=\frac{\mathrm{Y}_{\mathrm{SR}}}{2}\left(\mathrm{~V}_{\mathrm{S}_{1}}+\mathrm{V}_{\mathrm{R}_{1}} \mathrm{e}^{\mathrm{j} \delta}\right)
$$

Sendo $\delta$ o ângulo sincronização entre os dois terminais descritos.

A Equação 1 é complexa e pode ser desmembrada em duas equações reais, conforme mostrado nas Equações 2 e 3 .

$$
\begin{gathered}
\mathrm{I}_{\mathrm{S}_{1}} \cos \left(\theta_{\mathrm{I}_{1}}\right)+\mathrm{I}_{\mathrm{R}_{1}} \cos \left(\theta_{\mathrm{I}_{\mathrm{R}_{1}}}\right)=\frac{-\mathrm{b}_{\text {shunt }}}{2}\left(\mathrm{~V}_{\mathrm{S}_{1}} \operatorname{sen}\left(\theta_{\mathrm{V}_{\mathrm{S}_{1}}}\right)+\mathrm{V}_{\mathrm{R}_{1}} \operatorname{sen}\left(\theta_{\mathrm{V}_{\mathrm{R}_{1}}}+\delta\right)\right) \\
\mathrm{I}_{\mathrm{S}_{1}} \operatorname{sen}\left(\theta_{\mathrm{I}_{\mathrm{S}_{1}}}\right)+\mathrm{I}_{\mathrm{R}_{1}} \operatorname{sen}\left(\theta_{\mathrm{I}_{\mathrm{R}_{1}}}\right)=\frac{-\mathrm{b}_{\text {shunt }}}{2}\left(\mathrm{~V}_{\mathrm{S}_{1}} \cos \left(\theta_{\mathrm{V}_{\mathrm{S}_{1}}}\right)+\mathrm{V}_{\mathrm{R}_{1}} \cos \left(\theta_{\mathrm{V}_{\mathrm{R}_{1}}}+\delta\right)\right)
\end{gathered}
$$

Adotando:

$$
\mathrm{a}=\mathrm{I}_{\mathrm{S}_{1}} \cos \left(\theta_{\mathrm{I}_{\mathrm{S}_{1}}}\right)
$$




$$
\begin{aligned}
\mathrm{b}=\mathrm{I}_{\mathrm{S}_{1}} \operatorname{sen}\left(\theta_{\mathrm{I}_{\mathrm{S}_{1}}}\right) \\
\mathrm{c}=\mathrm{V}_{\mathrm{S}_{1}} \operatorname{sen}\left(\theta_{\mathrm{V}_{\mathrm{S}_{1}}}\right) \\
\mathrm{d}=\mathrm{V}_{\mathrm{S}_{1}} \cos \left(\theta_{\mathrm{V}_{\mathrm{S}_{1}}}\right)
\end{aligned}
$$

As Equações 2 e 3 podem ser reescritas conforme as Equações 8 e 9.

$$
\begin{aligned}
& \mathrm{a}+\mathrm{I}_{\mathrm{R}_{1}} \cos \left(\theta_{\mathrm{I}_{1}}+\delta\right)=\frac{-\mathrm{b}_{\text {shunt }}}{2}\left(\mathrm{c}+\mathrm{V}_{\mathrm{R}_{1}} \operatorname{sen}\left(\theta_{\mathrm{V}_{\mathrm{R}_{1}}}+\delta\right)\right) \\
& \mathrm{b}+\mathrm{I}_{\mathrm{R}_{1}} \cos \left(\theta_{\mathrm{I}_{1}}+\delta\right)=\frac{-\mathrm{b}_{\text {shunt }}}{2}\left(\mathrm{~d}+\mathrm{V}_{\mathrm{R}_{1}} \cos \left(\theta_{\mathrm{V}_{\mathrm{R}_{1}}}+\delta\right)\right)
\end{aligned}
$$

Agregando as Equações 8 e 9, obtém-se a Equação 10, que por sua vez gera a Equação 11 que depende somente de uma incógnita $\delta$.

$$
\begin{gathered}
\frac{\mathrm{a}+\mathrm{I}_{\mathrm{R}_{1}} \cos \left(\theta_{\mathrm{I}_{1}}+\delta\right)=\frac{-\mathrm{b}_{\text {shunt }}}{2}\left(\mathrm{c}+\mathrm{V}_{\mathrm{R}_{1}} \operatorname{sen}\left(\theta_{\mathrm{V}_{\mathrm{R}_{1}}}+\delta\right)\right)}{\mathrm{b}+\mathrm{I}_{\mathrm{R}_{1}} \cos \left(\theta_{\mathrm{I}_{1}}+\delta\right)=\frac{-\mathrm{b}_{\text {shunt }}}{2}\left(\mathrm{~d}+\mathrm{V}_{\mathrm{R}_{1}} \cos \left(\theta_{\mathrm{V}_{\mathrm{R}_{1}}}+\delta\right)\right)} \\
\mathrm{A}=\left(\mathrm{x}_{1}+\mathrm{x}_{2}+\mathrm{x}_{3}+\mathrm{x}_{4}\right) \cos (\delta)-\left(-\mathrm{y}_{1}+\mathrm{y}_{2}-\mathrm{y}_{3}+\mathrm{y}_{4}\right) \operatorname{sen}(\delta)
\end{gathered}
$$

Na Equação 11:

$$
\begin{gathered}
\mathrm{A}=\mathrm{ad}+\mathrm{bc}+\mathrm{V}_{\mathrm{R}_{1}} \mathrm{I}_{\mathrm{R}_{1}} \cos \left(\theta_{\mathrm{V}_{\mathrm{R}_{1}}}-\theta_{\mathrm{I}_{\mathrm{R}_{1}}}\right) \\
\mathrm{x}_{1}=-\mathrm{aV} \mathrm{V}_{\mathrm{R}_{1}} \cos \left(\theta_{\mathrm{V}_{\mathrm{R}_{1}}}\right) ; \mathrm{y}_{1}=\mathrm{aV}_{\mathrm{R}_{1}} \operatorname{sen}\left(\theta_{\mathrm{V}_{\mathrm{R}_{1}}}\right) \\
\mathrm{x}_{2}=-\mathrm{bV}_{\mathrm{R}_{1}} \operatorname{sen}\left(\theta_{\mathrm{V}_{\mathrm{R}_{1}}}\right) ; \mathrm{y}_{2}=\mathrm{bV}_{\mathrm{R}_{1}} \cos \left(\theta_{\mathrm{V}_{\mathrm{R}_{1}}}\right) \\
\mathrm{x}_{3}=-\mathrm{dI}_{\mathrm{R}_{1}} \cos \left(\theta_{\mathrm{I}_{1}}\right) ; \mathrm{y}_{3}=\mathrm{dI}_{\mathrm{R}_{1}} \operatorname{sen}\left(\theta_{\mathrm{I}_{\mathrm{R}_{1}}}\right) \\
\mathrm{x}_{4}=-\mathrm{cI}_{\mathrm{R}_{1}} \operatorname{sen}\left(\theta_{\mathrm{I}_{1}}\right) ; \mathrm{y}_{3}=\mathrm{cI}_{\mathrm{R}_{1}} \cos \left(\theta_{\mathrm{I}_{\mathrm{R}_{1}}}\right)
\end{gathered}
$$

Fazendo $\mathrm{x}=\mathrm{x}_{1}+\mathrm{x}_{2}+\mathrm{x}_{3}+\mathrm{x}_{4}$ e $\mathrm{y}=-\mathrm{y}_{1}+\mathrm{y}_{2}-\mathrm{y}_{3}+\mathrm{y}_{4}$ obtém-se a Equação 17.

$$
\mathrm{A}=\mathrm{x} \cos (\delta)-\mathrm{y} \operatorname{sen}(\delta)
$$

A partir da Equação 17 é possível representar a relação ente $A, x$ e $y$ por meio do triângulo retângulo, mostrado na Figura 2.

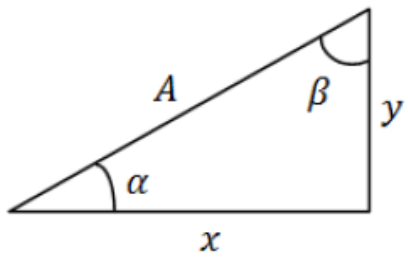

FIGURA 2. Relação geométrica ente A, y e x. [1]

A partir da análise conjunta da Equação 17 e da Figura 2, tem-se que: 


$$
\frac{\mathrm{A}}{\sqrt{\left(\mathrm{x}^{2}+\mathrm{y}^{2}\right)}}=\operatorname{sen}(\beta) \cos (\delta)-\operatorname{sen}(\delta) \cos (\beta)
$$

Dessa forma,

$$
\frac{\mathrm{A}}{\sqrt{\left(\mathrm{x}^{2}+\mathrm{y}^{2}\right)}}=\operatorname{sen}(\beta-\delta)
$$

A partir da Equação 19, pode-se determinar o ângulo de sincronização $\delta$, conforme a Equação 20.

$$
\delta=\operatorname{sen}^{-1}\left(\frac{x}{\sqrt{\left(x^{2}+y^{2)}\right.}}\right)-\operatorname{sen}^{-1}\left(\frac{A}{\sqrt{\left(x^{2}+y^{2}\right)}}\right)
$$

Considerando,

$$
\begin{aligned}
& \beta=\operatorname{sen}^{-1}\left(\frac{x}{\sqrt{\left(x^{2}+y^{2}\right)}}\right) \\
& \Phi=\operatorname{sen}^{-1}\left(\frac{A}{\sqrt{\left(x^{2}+y^{2}\right)}}\right)
\end{aligned}
$$

Assim,

$$
\delta=\beta-\Phi
$$

Em [1] para determinar o valor do ângulo $\delta$ no quadrante correto foi realizada uma análise sobre o sinal de $\mathrm{y}$, o que resultou no algoritmo a seguir.

$$
\begin{aligned}
& \text { Algoritmo: } \\
& \text { se }(y<0) \\
& \quad \delta=180^{\circ}-\beta-\Phi \\
& \text { senão } \\
& \quad \delta=\beta-\Phi \\
& \mathrm{fim}
\end{aligned}
$$

Para alguns casos de operação do sistema elétrico o algoritmo proposto em [1] expressou um ângulo de sincronização incorreto. Assim, [2] propôs uma nova metodologia, onde para se determinar o ângulo correto de sincronização é necessário considerar o módulo dos fasores de corrente em ambos os terminais, como mostrado a seguir.

$$
\begin{aligned}
& \text { Algoritmo: } \\
& \text { se }(x>0 \text { e } y>0) \\
& \text { se } I_{R_{1}}<I_{S_{1}} \\
& \delta=\beta_{1}-\Phi \\
& \text { fim } \\
& \text { se } I_{R_{1}}>I_{S_{1}} \\
& \delta=\beta_{1}-180^{\circ}+\Phi
\end{aligned}
$$




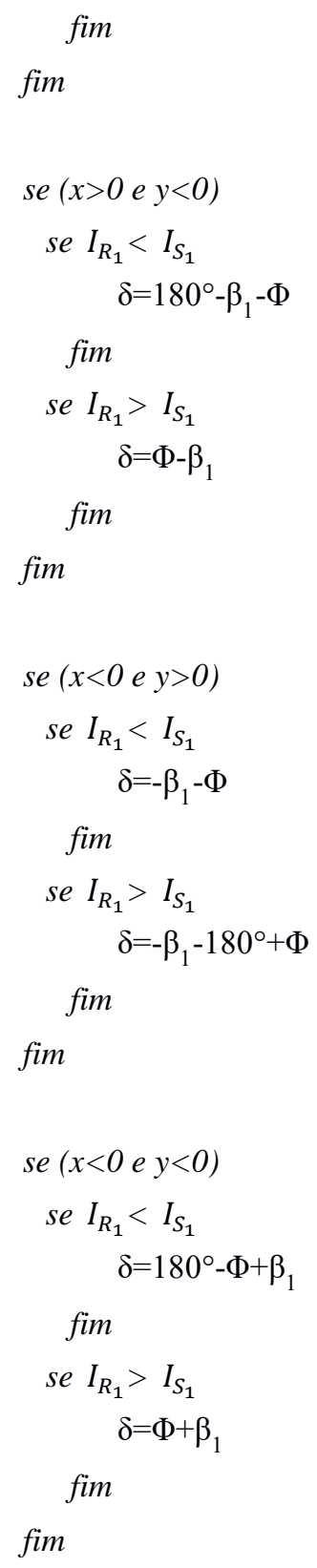

Vale salientar que $\beta_{1}$ no algoritmo acima é definido conforme a Equação 24.

$$
\beta_{1}=\operatorname{sen}^{-1}\left(\frac{|x|}{\sqrt{\left(x^{2}+y^{2}\right)}}\right)
$$

O algoritmo apresentado em [2] mostrou-se eficiente casos típicos de operação do sistema elétrico, determinando o ângulo de sincronização correto. Porém, ele possui um número elevado de equações e condições impostas para o sinal de $x$ que não existiam na metodologia descrita em [1].

Testes realizados no algoritmo proposto em [1] levaram ao novo algoritmo de sincronização proposto neste trabalho, que será apresentado na próxima seção. $\mathrm{O}$ algoritmo proposto é capaz de determinar o valor correto do ângulo de sincronização, para qualquer condição de operação do sistema, sem necessitar do conhecimento do sinal da variável $x$. Basicamente, conseguiu-se eliminar as limitações do algoritmo descrito em [1] apenas admitindo as condições do módulo de corrente descritas em [2].

Ressalta-se que em todas as metodologias de sincronização citadas neste trabalho, utiliza-se a Transformada Discreta de Fourier para a obtenção dos fasores de tensão e corrente pré-falta. 


\section{DEDUÇÃO DO ALGORITMO PROPOSTO}

Uma análise no algoritmo proposto em [1] foi efetuada para a dedução das equações e condições a serem utilizadas neste trabalho. Realizadas as simulações no ATPDraw e no ANAREDE, para determinadas situações de operação do sistema elétrico, foi analisado os valores absolutos das correntes $I_{R_{1}}$ e $I_{S_{1}}$, como também os sinais dos parâmetros x e y, e os ângulos $\beta$ e $\Phi$. Para isso foram escolhidos ângulos de sincronização de $120^{\circ} \mathrm{a}$ $-120^{\circ}$. As Tabelas 1,2 e 3 apresentam estes parâmetros para simulação de uma LT de $230 \mathrm{kV}$ considerando as situações de $0,95 \mathrm{pu}, 1 \mathrm{pu}$ e 1,05 pu no terminal $\mathrm{V}_{\mathrm{R}}$. As Tabelas 4 e 5 expressam os mesmos parâmetros para umaa $\mathrm{LT}$ real de $230 \mathrm{kV}$ e $500 \mathrm{kV}$, simulada no ANAREDE.

TABELA 1. Parâmetros e valores analisados para $\mathrm{V}_{\mathrm{R}}$ igual a 0,95 pu. (Autoria própria)

\begin{tabular}{|c|c|c|c|c|c|c|c|}
\hline \multicolumn{8}{|c|}{ Tensão 0,95 pu } \\
\hline \multirow{2}{*}{$\begin{array}{r}\hat{A} \text { ngulo de } \\
\text { sincronização } \\
\text { real (graus) }\end{array}$} & \multicolumn{6}{|c|}{ Parâmetros } & \multirow{2}{*}{$\begin{array}{c}\hat{A} \text { Angulo de } \\
\text { sincronização } \\
\text { calculado } \\
\text { (graus) }\end{array}$} \\
\hline & $\begin{array}{c}\beta \\
\text { (graus) }\end{array}$ & $\begin{array}{c}\Phi \\
\text { (graus) }\end{array}$ & $x$ & $y$ & $I_{R_{1}}(A)$ & $I_{S_{1}}(A)$ & \\
\hline 120 & $\begin{array}{c}- \\
66,2545 \\
\end{array}$ & 6,5293 & - & + & 296,5176 & 285,0606 & $-72,79$ \\
\hline 90 & $\begin{array}{c}- \\
83,7455\end{array}$ & 6,5293 & - & - & 296,5176 & 285,0606 & 257,21 \\
\hline 60 & $\begin{array}{c}- \\
53,7455 \\
\end{array}$ & 6,5293 & - & - & 296,5176 & 285,0606 & 227,21 \\
\hline 30 & $\begin{array}{c}- \\
23,7475 \\
\end{array}$ & 6,5293 & - & - & 296,5176 & 285,0606 & 197,21 \\
\hline 0 & 6,2545 & 6,5293 & + & - & 296,5176 & 285,0606 & 167,21 \\
\hline-30 & 36,2545 & 6,5293 & + & - & 296,5176 & 285,0606 & 137,21 \\
\hline-60 & 66,2545 & 6,5293 & + & - & 296,5176 & 285,0606 & 107,21 \\
\hline-90 & 83,7455 & 6,5293 & + & + & 296,5176 & 285,0606 & 77,21 \\
\hline-120 & 53,7455 & 6,5293 & + & + & 296,5176 & 285,0606 & 47,21 \\
\hline
\end{tabular}

TABELA 2. Parâmetros e valores analisados para $\mathrm{V}_{\mathrm{R}}$ igual a 1 pu. (Autoria própria)

\begin{tabular}{ccccccccc}
\hline \multicolumn{7}{c}{ Tensão l pu } \\
\hline $\begin{array}{c}\text { Anngulo de } \\
\text { sincronização } \\
\text { real (graus) }\end{array}$ & \begin{tabular}{c}
$\beta$ \\
\cline { 2 - 8 }
\end{tabular} & $\begin{array}{c}\text { Praumetros } \\
\text { (graus) }\end{array}$ & $x$ & $y$ & $I_{R_{1}}(A)$ & $I_{S_{1}}(A)$ & $\begin{array}{c}\text { Angulo de } \\
\text { sincronização } \\
\text { calculado } \\
\text { (graus) }\end{array}$ \\
\hline 120 & 56,0214 & 4,1682 & + & - & 277,1477 & 295,8820 & 119,81 \\
\hline 90 & 86,0214 & 4,1682 & + & - & 277,1477 & 295,8820 & 89,81 \\
\hline 60 & 63,9786 & 4,1682 & + & + & 277,1477 & 295,8820 & 59,81 \\
\hline 30 & 33,9786 & 4,1682 & + & + & 277,1477 & 295,8820 & 29,81 \\
\hline 0 & 3,9786 & 4,1682 & + & + & 277,1477 & 295,8820 & $-0,19$ \\
\hline-30 & $-26,0414$ & 4,1682 & - & + & 277,1477 & 295,8820 & $-30,19$ \\
\hline-60 & $-56,0214$ & 4,1682 & - & + & 277,1477 & 295,8820 & $-60,19$ \\
\hline-90 & $-86,214$ & 4,1682 & - & + & 277,1477 & 295,8820 & $-90,19$ \\
\hline-120 & $-63,9786$ & 4,1682 & - & - & 277,1477 & 295,8820 & 239,81 \\
\hline
\end{tabular}


TABELA 3. Parâmetros e valores analisados para $\mathrm{V}_{\mathrm{R}}$ igual a 1,05 pu. (Autoria própria)

\begin{tabular}{|c|c|c|c|c|c|c|c|}
\hline \multicolumn{8}{|c|}{ Tensão 1,05 pu } \\
\hline \multirow{2}{*}{$\begin{array}{l}\hat{A} n g u l o d e \\
\text { sincronização } \\
\text { real (graus) }\end{array}$} & \multicolumn{6}{|c|}{ Parâmetros } & \multirow{2}{*}{$\begin{array}{l}\hat{A} \text { Angulo de } \\
\text { sincronização } \\
\text { calculado } \\
\text { (graus) }\end{array}$} \\
\hline & $\beta$ (graus) & $\Phi$ (graus) & $x$ & $y$ & $I_{R_{1}}(A)$ & $I_{S_{1}}(A)$ & \\
\hline 120 & 58,3499 & 1,7228 & + & - & 279,8393 & 327,5798 & 119,95 \\
\hline 90 & 88,3499 & 1,7228 & + & - & 279,8393 & 327,5798 & 89,95 \\
\hline 60 & 61,6501 & 1,7228 & + & + & 279,8393 & 327,5798 & 59,95 \\
\hline 30 & 31,6501 & 1,7228 & + & + & 279,8393 & 327,5798 & 29,95 \\
\hline 0 & 1,6501 & 1,7228 & + & + & 279,8393 & 327,5798 & 0,045 \\
\hline-30 & $-28,3499$ & 1,7228 & - & + & 279,8393 & 327,5798 & $-30,05$ \\
\hline-60 & $-58,3499$ & 1,7228 & - & + & 279,8393 & 327,5798 & $-60,05$ \\
\hline-90 & $-88,3499$ & 1,7228 & - & + & 279,8393 & 327,5798 & $-90,05$ \\
\hline-120 & $-61,6501$ & 1,7228 & - & - & 279,8393 & 327,5798 & 239,95 \\
\hline
\end{tabular}

Assim percebeu-se que os casos onde o código proposto em [1] funciona quando os valores de $I_{S_{1}}$ foram maiores que os de $I_{R_{1}}$, porém isso não ocorre em casos contrários. A partir da análise dos dados, é possível concluir que isso ocorreu devido o sinal do ângulo $\beta$, para cada ângulo de sincronização, se inverter quando o valor de $I_{S_{1}}$ é menor que os de $I_{R_{1}}$. Dessa forma, é necessário analisar, além do sinal de y, também a grandeza dos valores absolutos de corrente pré-falta em ambos os terminais, como relatado em [2].

Diante disso, para a determinação do novo código foram preservadas as equações utilizadas pela metodologia proposta em [1], com o acréscimo de duas equações contendo as condições dos valores absolutos de corrente pré-falta em ambos os terminais.

Realizou-se esta mesma análise para as LTs de 230 e $500 \mathrm{kV}$, simuladas no software ANAREDE, onde notou-se que o padrão visto nos casos anteriores, se repete. Observa-se isso nas Tabelas 4 e 5.

TABELA 4. Parâmetros e valores analisados para LT real de 230 kV. (Autoria própria)

\begin{tabular}{|c|c|c|c|c|c|c|c|}
\hline \multicolumn{8}{|c|}{ Tensão $230 \mathrm{kV}$} \\
\hline \multirow{2}{*}{$\begin{array}{l}\text { Ângulo de } \\
\text { sincronização } \\
\text { real (graus) }\end{array}$} & \multicolumn{6}{|c|}{ Parâmetros } & \multirow{2}{*}{$\begin{array}{c}\text { Ângulo de } \\
\text { sincronização } \\
\text { calculado } \\
\text { (graus) }\end{array}$} \\
\hline & $\begin{array}{c}\beta \\
\text { (graus) }\end{array}$ & $\begin{array}{c}\Phi \\
\text { (graus) }\end{array}$ & $x$ & $y$ & $\begin{array}{c}I_{R_{1}} \\
(p u)\end{array}$ & $\begin{array}{c}I_{S_{1}} \\
(p u)\end{array}$ & \\
\hline 120 & 59,9198 & $-0,0494$ & + & - & 0,2500 & 0,2700 & 120,04 \\
\hline 90 & 89,9198 & $-0,0494$ & + & - & 0,2500 & 0,2700 & 90,04 \\
\hline 60 & 60,0802 & $-0,0494$ & + & + & 0,2500 & 0,2700 & 60,04 \\
\hline 30 & 30,0802 & $-0,0494$ & + & + & 0,2500 & 0,2700 & 30,04 \\
\hline 0 & 0,0802 & $-0,0494$ & + & + & 0,2500 & 0,2700 & 0,04 \\
\hline-30 & $-29,9198$ & $-0,0494$ & - & + & 0,2500 & 0,2700 & $-29,96$ \\
\hline-60 & $-59,9198$ & $-0,0494$ & - & + & 0,2500 & 0,2700 & $-59,96$ \\
\hline-90 & $-89,9198$ & $-0,0494$ & - & + & 0,2500 & 0,2700 & $-89,96$ \\
\hline-120 & $-60,0802$ & $-0,0494$ & - & - & 0,2500 & 0,2700 & 240,04 \\
\hline
\end{tabular}


TABELA 5. Parâmetros e valores analisados para LT real de 500 kV. (Autoria própria)

\begin{tabular}{|c|c|c|c|c|c|c|c|}
\hline \multicolumn{8}{|c|}{ Tensão 500kV } \\
\hline \multirow{2}{*}{$\begin{array}{l}\hat{A} \text { Angulo de } \\
\text { sincronização } \\
\text { real (graus) }\end{array}$} & \multicolumn{6}{|c|}{ Parâmetros } & \multirow{2}{*}{$\begin{array}{c}\text { Ângulo de } \\
\text { sincronização } \\
\text { calculado } \\
\text { (graus) }\end{array}$} \\
\hline & $\begin{array}{c}\beta \\
(\text { graus })\end{array}$ & $\begin{array}{c}\Phi \\
(\text { graus })\end{array}$ & $x$ & $y$ & $\begin{array}{c}I_{R_{1}} \\
(p u)\end{array}$ & $\begin{array}{c}I_{S_{1}} \\
(p u)\end{array}$ & \\
\hline 120 & $-60,1998$ & 0,0357 & - & + & 1,8000 & 1,3000 & $-60,24$ \\
\hline 90 & $-89,8002$ & 0,0357 & - & - & 1,8000 & 1,3000 & 269,76 \\
\hline 60 & $-59,8002$ & 0,0357 & - & - & 1,8000 & 1,3000 & 239,76 \\
\hline 30 & $-29,8002$ & 0,0357 & - & - & 1,8000 & 1,3000 & 209,76 \\
\hline 0 & 0,1998 & 0,0357 & + & - & 1,8000 & 1,3000 & 179,76 \\
\hline-30 & 30,1998 & 0,0357 & + & - & 1,8000 & 1,3000 & 149,76 \\
\hline-60 & 60,1998 & 0,0357 & + & - & 1,8000 & 1,3000 & 119,76 \\
\hline-90 & 89,8002 & 0,0357 & + & + & 1,8000 & 1,3000 & 89,76 \\
\hline-120 & 59,8002 & 0,0357 & + & + & 1,8000 & 1,3000 & 59,76 \\
\hline
\end{tabular}

A partir da análise destes parâmetros, nestas situações de operação do sistema elétrico, elaborou-se o algoritmo proposto neste trabalho, mostrado abaixo.

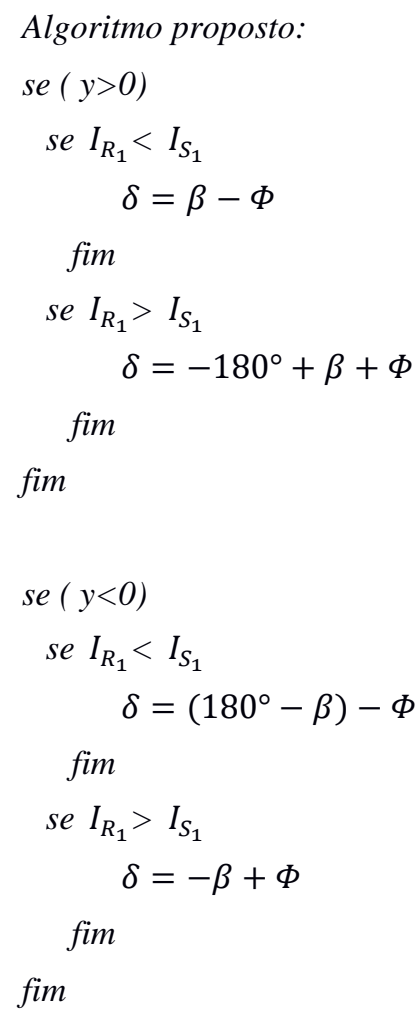

\section{SISTEMA ANALISADO}

Para a análise de desempenho do algoritmo proposto, foram realizadas simulações no software ATPDraw, para sistemas elétricos fictícios e no software ANAREDE para sistemas reais.

A Figura 3 ilustra o sistema elétrico modelado no ATPDraw para os níveis de tensão de 230 e $500 \mathrm{kV}$. A linha de transmissão 2 (LT2), possui $180 \mathrm{~km}$ de comprimento para a tensão de $230 \mathrm{kV}$ e $250 \mathrm{~km}$ para $500 \mathrm{kV}$, sendo em seus terminais onde são coletados os fasores de tensão e corrente. As linhas de transmissão 1 e 3 (LT1 e LT3) tem $20 \mathrm{~km}$ de comprimento nos dois níveis de tensão. Estas simulações foram realizadas considerando 
linhas de transmissão transpostas, utilizando a modelagem de parâmetros distribuídos. Os parâmetros de sequência positiva e zero das linhas são mostrados nas Tabelas 6 e 7.

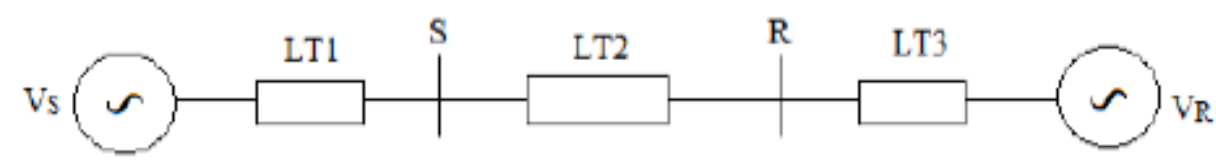

FIGURA 3. Representação unifilar da LT. [2]

TABELA 6. Parâmetros de sequência positiva e zero da linha de 230 kV. (Autoria própria)

\begin{tabular}{cccc}
\hline \multicolumn{4}{c}{ Tensão $230 \mathrm{kV}$} \\
\hline Sequência & $R(\Omega / \mathrm{km})$ & $L(\mathrm{mH} / \mathrm{km})$ & $C(\mu \mathrm{F} / \mathrm{km})$ \\
\hline Positiva & 0,09127 & 1,376 & 0,00847 \\
\hline Zero & 0,44016 & 4,1538 & 0,00610 \\
\hline
\end{tabular}

TABELA 7. Parâmetros de sequência positiva e zero da linha de 500 kV. (Autoria própria)

\begin{tabular}{cccc}
\hline \multicolumn{4}{c}{ Tensão $500 \mathrm{kV}$} \\
\hline Sequência & $R(\Omega / \mathrm{km})$ & $L(\mathrm{mH} / \mathrm{km})$ & $C(\mu F / \mathrm{km})$ \\
\hline Positiva & 0,0221 & 0,852 & 0,01346 \\
\hline Zero & 0,3777 & 3,7767 & 0,00858 \\
\hline
\end{tabular}

Para cada nível de tensão simulado no ATPDraw, foram testadas três situações de operação do sistema elétrico como pode ser visto na Tabela 8. Foi considerado em todos os casos a fase de $V_{S}$ igual a $0^{\circ}$ e $V_{R}$ igual a $-10^{\circ}$. Em todas as simulações foram utilizadas uma taxa de 256 amostras por ciclo.

TABELA 8. Situações analisadas do sistema elétrico. (Autoria própria)

\begin{tabular}{cccc}
\hline \multicolumn{4}{c}{ Situações de operação do sistema elétrico } \\
\hline Parâmetro & \multicolumn{3}{c}{ Tensão $($ pu $)$} \\
\hline $\mathrm{V}_{\mathrm{S}}$ & 1 & 1 & 1 \\
\hline $\mathrm{V}_{\mathrm{R}}$ & 0,95 & 1 & 1,05 \\
\hline
\end{tabular}

No software ANAREDE simulou-se sistemas elétricos reais para 230 e $500 \mathrm{kV}$. Para $230 \mathrm{kV}$ foi simulada a linha de transmissão Natal II (5241) - Natal III (5245) com 11,55 km de comprimento. Já para o nível de tensão de $500 \mathrm{kV}$ foi simulada a linha de Transmissão Luiz Gonzaga (5050) - Milagres (5408) com 231,2 km de comprimento. Na Tabela 9 são mostrados os parâmetros de sequência positiva para as linhas de transmissão de 230 e $500 \mathrm{kV}$.

TABELA 9. Parâmetros de sequência positiva - LTs simuladas no ANAREDE. (Autoria própria)

\begin{tabular}{cccc}
\hline \multicolumn{4}{c}{ Parâmetros sequência positiva } \\
\hline Tensão $(k V)$ & $R(\Omega / \mathrm{km})$ & $L(\mathrm{mH} / \mathrm{km})$ & $C(\mu \mathrm{F} / \mathrm{km})$ \\
\hline 230 & 0,0504 & 0,935 & 0,00866 \\
\hline 500 & 0,0238 & 0,746 & 0,016
\end{tabular}

\section{RESUltAdOS}

Com base nas simulações realizadas nos softwares ATPDraw e ANAREDE, este tópico expõe os resultados avaliativos do algoritmo de sincronização proposto. Entretanto, os valores de tensão oriundos desses softwares são sincronizados automaticamente por eles, sendo necessário à realização de uma dessincronização artificial.

Para uma melhor avaliação do algoritmo de sincronização apontado, foram inseridos erros de sincronização nos fasores de tensão e corrente nos terminais R, iguais aos utilizados em [2] para uma melhor comparação dos dados. 
Na Tabela 10 são expostos os ângulos de sincronização calculados pela metodologia relatada neste artigo, como também pelos algoritmos demonstrados em [1] e [2]. Isto para as linhas de transmissão de 230 e $500 \mathrm{kV}$ simuladas no ATPDraw, para a condição de tensão de 0,95 pu no terminal $\mathrm{R}$.

TABELA 10. Ângulos calculados para situação de operação de 0,95 pu do sistema, para LTs de 230 e 500 $\mathrm{kV}$ (Autoria própria)

\begin{tabular}{|c|c|c|c|c|c|c|}
\hline \multirow{3}{*}{$\begin{array}{l}\text { Ângulo de } \\
\text { sincronização } \\
\text { real (graus) }\end{array}$} & \multicolumn{6}{|c|}{ Ângulo de sincronização calculado (graus) } \\
\hline & \multicolumn{2}{|c|}{ Algoritmo proposto } & \multicolumn{2}{|c|}{$\begin{array}{c}\text { Algoritmo proposto em } \\
{[1]}\end{array}$} & \multicolumn{2}{|c|}{$\begin{array}{c}\text { Algoritmo proposto em } \\
{[2]}\end{array}$} \\
\hline & $230 \mathrm{kV}$ & $500 \mathrm{kV}$ & $230 \mathrm{kV}$ & $500 \mathrm{kV}$ & $230 \mathrm{kV}$ & $500 \mathrm{kV}$ \\
\hline 120 & $-239,72$ & $-239,85$ & $-72,79$ & $-62,77$ & $-239,73$ & $-239,85$ \\
\hline 90 & 90,27 & 90,15 & 257,21 & 267,23 & 90,27 & 90,15 \\
\hline 60 & 60,27 & 60,15 & 227,21 & 237,23 & 60,27 & 60,15 \\
\hline 30 & 30,27 & 30,15 & 197,21 & 207,23 & 30,27 & 30,15 \\
\hline 0 & 0,27 & 0,15 & 167,21 & 177,23 & 0,27 & 0,15 \\
\hline-30 & $-29,73$ & $-29,85$ & 137,21 & 147,23 & $-29,73$ & $-29,85$ \\
\hline-60 & $-59,73$ & $-59,85$ & 107,21 & 117,23 & $-60,73$ & $-59,85$ \\
\hline-90 & $-89,73$ & $-89,85$ & 77,21 & 87,23 & $-89,73$ & $-89,85$ \\
\hline-120 & $-119,73$ & $-119,85$ & 47,21 & 57,23 & $-119,73$ & $-119,85$ \\
\hline
\end{tabular}

Nota-se que o algoritmo mostrado em [1] apresentou valores incorretos para ambos os níveis de tensão. Para o algoritmo deste estudo, foram encontrados valores corretos de ângulos de sincronização, estes bem parecidos com os encontrados pela metodologia realizada em [2].

Os ângulos de sincronização calculados não são exatos devido às margens de erro decorrentes das aproximações numéricas realizadas pelo algoritmo. Contudo, esta margem de erro é bem pequena, sendo que para o algoritmo proposto ela é de $0,27^{\circ}$ para a tensão de $230 \mathrm{kV}$ e de $0,15^{\circ}$ para a tensão de $500 \mathrm{kV}$.

Ainda utilizando os dados das simulações realizadas no ATPDraw, as Tabelas 11 e 12 trazem os ângulos de sincronização calculados para os níveis de 1 pu e 1,05 pu no terminal R.

TABELA 11. Ângulos calculados para situação de operação de 1 pu do sistema, para LTs de 230 e 500 kV (Autoria própria)

\begin{tabular}{|c|c|c|c|c|c|c|}
\hline \multirow{3}{*}{$\begin{array}{c}\hat{A} \text { ngulo de } \\
\text { sincronização } \\
\text { real (graus) }\end{array}$} & \multicolumn{6}{|c|}{ Ângulo de sincronização calculado (graus) } \\
\hline & \multicolumn{2}{|c|}{ Algoritmo proposto } & \multicolumn{2}{|c|}{$\begin{array}{c}\text { Algoritmo proposto em } \\
{[1]}\end{array}$} & \multicolumn{2}{|c|}{$\begin{array}{c}\text { Algoritmo proposto em } \\
{[2]}\end{array}$} \\
\hline & $230 \mathrm{kV}$ & $500 \mathrm{kV}$ & $230 \mathrm{kV}$ & $500 \mathrm{kV}$ & $230 \mathrm{kV}$ & $500 \mathrm{kV}$ \\
\hline 120 & 119,81 & 119,50 & 119,81 & 119,50 & 119,81 & 119,50 \\
\hline 90 & 89,81 & 89,50 & 89,81 & 89,50 & 89,81 & 89,50 \\
\hline 60 & 59,81 & 59,50 & 59,81 & 60,50 & 59,81 & 60,50 \\
\hline 30 & 29,81 & 29,50 & 29,81 & 29,50 & 29,81 & 29,50 \\
\hline 0 & $-0,19$ & $-0,50$ & $-0,19$ & 0,50 & $-0,19$ & 0,50 \\
\hline-30 & $-30,19$ & $-30,50$ & $-30,19$ & $-30,50$ & $-30,19$ & $-30,50$ \\
\hline-60 & $-60,19$ & $-60,50$ & $-60,19$ & $-60,50$ & $-60,19$ & $-60,50$ \\
\hline-90 & $-90,19$ & $-90,50$ & $-90,19$ & $-90,50$ & $-90,19$ & $-90,50$ \\
\hline-120 & 239,81 & 239,50 & 239,81 & 239,50 & 239,81 & 239,50 \\
\hline
\end{tabular}


TABELA 12. Ângulos calculados para situação de operação de 1,05 pu do sistema, para LTs de 230 e 500 $\mathrm{kV}$ (Autoria própria)

\begin{tabular}{ccccccc}
\hline \multirow{2}{*}{$\begin{array}{c}\text { Ângulo de } \\
\text { sincronização } \\
\text { real }(\text { graus) }\end{array}$} & Algoritmo proposto & Algoritmo proposto em & Algoritmo proposto em \\
\cline { 2 - 7 } & $230 \mathrm{kV}$ & $500 \mathrm{kV}$ & $230 \mathrm{kV}$ & $500 \mathrm{kV}$ & $230 \mathrm{kV}$ & $500 \mathrm{kV}$ \\
\hline 120 & 119,93 & 119,90 & 119,95 & 119,90 & 119,95 & 119,90 \\
\hline 90 & 89,93 & 89,90 & 89,95 & 89,90 & 89,95 & 89,90 \\
\hline 60 & 59,93 & 59,90 & 59,95 & 59,90 & 59,95 & 59,90 \\
\hline 30 & 29,93 & 29,90 & 29,95 & 29,90 & 29,95 & 29,90 \\
\hline 0 & $-0,07$ & $-0,10$ & 0,045 & 0,10 & 0,045 & 0,10 \\
\hline-30 & $-30,07$ & $-30,10$ & $-30,05$ & $-30,10$ & $-30,05$ & $-30,10$ \\
\hline-60 & $-60,07$ & $-60,10$ & $-60,05$ & $-60,10$ & $-60,05$ & $-60,10$ \\
\hline-90 & $-90,07$ & $-90,10$ & $-90,05$ & $-90,10$ & $-90,05$ & $-90,10$ \\
\hline-120 & 239,93 & 239,90 & 239,95 & 239,90 & 239,95 & 239,90 \\
\hline
\end{tabular}

Para estas condições, o algoritmo proposto neste trabalho, como também os colocados em [1] e em [2] apresentaram valores de ângulos de sincronização aproximados. Para a condição de 1 pu, foram encontrados erros absolutos de $0,19^{\circ}$ para a tensão de $230 \mathrm{kV}$ e de $0,5^{\circ}$ para $500 \mathrm{kV}$. Já o erro absoluto encontrado para a condição de 1,05 pu foi de $0,07^{\circ}$ para a tensão de $230 \mathrm{kV}$ e de $0,1^{\circ}$ para a tensão de $500 \mathrm{kV}$.

$\mathrm{Na}$ Tabela 13 podem-se observar os valores dos ângulos de sincronização calculados pelo algoritmo desenvolvido nesse estudo, e também em [1] e [2], para os casos de sistemas reais simulados no ANAREDE com linhas de transmissão de 230 e 500 kV.

TABELA 13. Ângulos calculados para sistemas reais simulados no ANAREDE, para LTs de 230 e $500 \mathrm{kV}$ (Autoria própria)

\begin{tabular}{|c|c|c|c|c|c|c|}
\hline \multirow{3}{*}{$\begin{array}{l}\hat{A} \text { Angulo de } \\
\text { sincronização } \\
\text { real (graus) }\end{array}$} & \multicolumn{6}{|c|}{ Ângulo de sincronização calculado (graus) } \\
\hline & \multicolumn{2}{|c|}{ Algoritmo proposto } & \multicolumn{2}{|c|}{$\begin{array}{c}\text { Algoritmo proposto } \\
\text { em }[1]\end{array}$} & \multicolumn{2}{|c|}{$\begin{array}{c}\text { Algoritmo proposto } \\
\text { em [2] }\end{array}$} \\
\hline & $230 \mathrm{kV}$ & $500 \mathrm{kV}$ & $230 k \mathrm{~V}$ & $500 \mathrm{kV}$ & $230 \mathrm{kV}$ & $500 \mathrm{kV}$ \\
\hline 120 & 120,13 & 119,84 & 120,04 & $-60,24$ & 120,04 & $-240,17$ \\
\hline 90 & 90,13 & 89,85 & 90,04 & 269,76 & 90,04 & 89,83 \\
\hline 60 & 60,13 & 59,85 & 60,04 & 239,76 & 60,04 & 59,83 \\
\hline 30 & 30,13 & 29,85 & 30,04 & 209,76 & 30,04 & 29,83 \\
\hline 0 & 0,13 & $-0,16$ & 0,04 & 179,76 & 0,04 & 0,17 \\
\hline-30 & $-29,87$ & $-30,16$ & $-29,96$ & 149,76 & $-29,96$ & $-30,17$ \\
\hline-60 & $-59,87$ & $-60,16$ & $-59,96$ & 119,76 & $-59,96$ & $-60,17$ \\
\hline-90 & $-89,87$ & 269,84 & $-89,96$ & 89,76 & $-89,96$ & $-90,17$ \\
\hline-120 & 240,13 & 239,84 & 240,04 & 59,76 & 240,04 & $-120,17$ \\
\hline
\end{tabular}

É notório que para a tensão de $230 \mathrm{kV}$, todos os algoritmos sugeridos apresentaram ângulos de sincronização corretos, cada um com sua margem de erro respectiva. Todavia, para tensão de $500 \mathrm{kV}$, o código proposto em [1] apresentou valores imprecisos, enquanto que o código aprimorado neste trabalho e o desenvolvido por [2] obtiveram valores de ângulos de sincronização mais precisos. A metodologia proposta nesse artigo apontou erro absoluto de apenas $0,13^{\circ}$ para a tensão de $230 \mathrm{kV}$ e de $0,16^{\circ}$ para tensão de $500 \mathrm{kV}$.

\section{CONCLUSÃO}

R4EM, v.1, n.1, p. 100-111, 2019 
A partir do estudo realizado neste trabalho foi possível obter um novo equacionamento para a sincronização de registros, sendo uma versão aperfeiçoada das metodologias propostas em [1] e [2]. Ou seja, conseguiu-se eliminar as limitações do algoritmo proposto em [1], fundamentando-se no uso das condições das correntes terminais, proposta em [2]. Com isso, obteve-se uma eficiente ferramenta de sincronização que funciona com um número reduzido de equações, para qualquer situação do sistema elétrico. Além disso, preserva a característica de ser independente dos parâmetros elétricos da linha, sendo dependente unicamente dos fasores de tensão e corrente pré-falta.

\section{REFERÊNCIAS}

[1] CRUZ, Melinda Cesianara Silva da; OLIVEIRA, José Tavares de; MEDEIROS JUNIOR, Manoel Firmino de. Sincronização de Registros Oscilográficos em Linhas de Transmissão com Parâmetros Elétricos Desconhecidos. In: SIMPÓSIO BRASILEIRO DE SISTEMAS ELÉTRICOS, 6., 2016, Natal. Anais... . Natal: Simpósio Brasileiro de Sistemas Elétricos, 2016. p. 1 - 4.

[2] MONTEIRO, Roana D. S. et al. Um novo algoritmo para sincronização de Registros Oscilográficos em Linhas de Transmissão com Parâmetros Elétricos Desconhecidos. XXII Congresso Brasileiro de Automática, João Pessoa, 2018. p. $1-7$.

[3] CRUZ, M. C. S. da, Almeida, M. A. D., Oliveira, J. T., Cândido, C. K. S. S., Leitão, J. J. A. L., Medeiros Jr, M. F. (2010). Localização de Faltas em Linhas de Transmissão de Múltiplos Terminais a Partir de Registros Oscilográficos Sincronizados Via Transformada Wavelet. In: IEEE/PES Transmission \& Distribution Conference and Exposition Latin America.

[4] COURY, Denis Vinicius; OLESKOVICZ, Mário; GIOVANINI, Renan. Proteção Digital de Sistemas Elétricos de Potência: dos relés eletromecânicos aos microprocessados inteligentes. São Carlos-sp: Eesc-usp, 2007. $378 \mathrm{p}$.

[5] CRUZ, Melinda Cesianara Souza et al. Metodologia para Sincronização de Registros de Falta baseada em Equações de Circuito Equivalente de Linhas de Transmissão. Ieee/ias International Conference On Industry Applications, Fortaleza, v. 10, n. 10, p.120-126, fev. 2012

[6] Y. Liao e N. Kang, "Fault-Location Algorithms Without utilizing Line Parameters Based on the Distributed Parameter Line Model”, IEEE Trans. Power Deliv., vol. 24, no. 2, p. 579-584, 2009. 